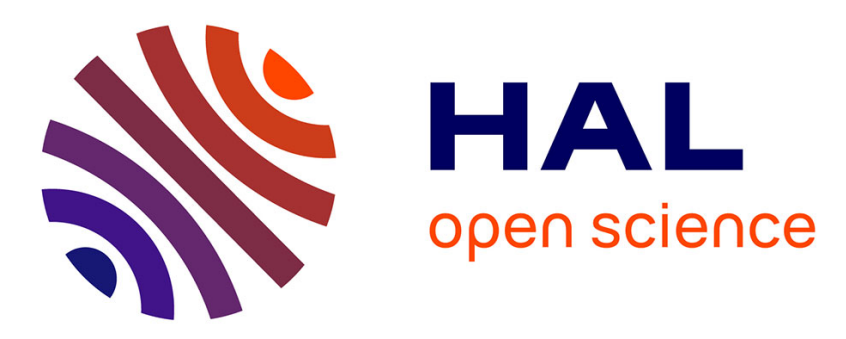

\title{
From restricted isometry property to observability under sparse measurement
}

Yassine Y. Khaled Khaled, Jean-Pierre Barbot, Krishna Busawon, Djamila

Benmerzouk

\section{- To cite this version:}

Yassine Y. Khaled Khaled, Jean-Pierre Barbot, Krishna Busawon, Djamila Benmerzouk. From restricted isometry property to observability under sparse measurement. International Conference on Systems and Control, Oct 2013, Alger, Algeria. hal-00923629

\section{HAL Id: hal-00923629 \\ https://hal.inria.fr/hal-00923629}

Submitted on 3 Jan 2014

HAL is a multi-disciplinary open access archive for the deposit and dissemination of scientific research documents, whether they are published or not. The documents may come from teaching and research institutions in France or abroad, or from public or private research centers.
L'archive ouverte pluridisciplinaire HAL, est destinée au dépôt et à la diffusion de documents scientifiques de niveau recherche, publiés ou non, émanant des établissements d'enseignement et de recherche français ou étrangers, des laboratoires publics ou privés. 


\title{
From restricted isometry property to observability under sparse measurement
}

\author{
Y. Khaled ${ }^{1,2}$, J-P. Barbot ${ }^{1,3}$, K. Busawon ${ }^{4}$ and D. Benmerzouk ${ }^{2}$ \\ ${ }^{1}$ ECS-Lab ENSEA, 6 Avenue du Ponceau, 95014 Cergy-Pontoise, France \\ ${ }^{2}$ Department of Mathematics, Tlemcen University, Algeria \\ ${ }^{3}$ EPI Non-A, INRIA Lille, Nord-Europe \\ ${ }^{4}$ Northumbria University, Faculty of Engineering and Environment, Newcastle upon Tyne, UK
}

\begin{abstract}
In this paper, we show that it is possible to detect the active mode and reconstruct its associated state for a class of hybrid linear systems under sparse measurements. The solution we bring to this problem is firstly to analyze the observability of systems under random sampling and secondly to synthesize a impulsive observer. Here, the first approach is based on the concept of compressive sampling which is well-known in signal processing theory. A synthesis of impulsive observers will presented for some special cases. These observers allows to detect the active mode and rebuild the state under sparse measurement. Simulation results are provided in order to highlight the wellfoundedness of the proposed approach.
\end{abstract}

Keywords: Hybrid system, impulsive observer, compressive sensing

\section{INTRODUCTION}

In the theory of signal processing, it is well known that, under certain circumstances, it is possible to reconstruct a signal even if the sampling frequency is less than the Nyquist frequency(see eg. [10] and [4]). In fact, compressive sampling (CS) is based on the hypothesis that the signal is located in an extended space that is reconstructed in a suitable base [22] and the associated matrix verifies a restricted isometry property (RIP)[3].

Under these assumptions, the signal can be reconstructed using techniques based on regularised linear regression. This results naturally leads one to ask if it is possible to bypass the Nyquist-Shannon sampling frequency constraint in a closedloop control. This question, in turn, generates several essential ones:

i) How can we translate (CS) from a signal theory context into the dynamical system theory context?

ii) What is the appropriate base in a dynamical system context? iii) How can one verify the (RIP) property in a dynamical system context?

iv) How can one bypass the optimization algorithm (generally carried out off-line) in order to cope with real-time algorithms? v) How can one guarantee the closed-loop stability under sparse measurements?

An answer to the question i) is partially given in signal processing literature. As in [23], in this work a model based on (CS) is presented, even though this type of model is very different compared to usual dynamical systems. An answer to the question ii) is implicitly given several articles on observation and diagnosis of dynamical systems [1], [2]. Specifically, a suitable base is the base of the normal form [12] associated with the Whitney topology together with all the restrictive considerations with respect to genericity and the sensitivity of parameters [11].

In this paper, we will answer to the question iii). More precisely, we propose a sufficient condition related to the observability and distinguishability of hybrid systems that will allows us to detect and reconstruct the non-measured states of a continuous linear system with sparse measurements.

An answer to the question iv) for linear systems is given in [13] and for chaotic systems in [14] and will be used here to illustrate the answer to the question v). More precisely, a impulsive observer which bypasses the optimization algorithm, under certain conditions, will be presented in this work.

This paper is organized as follows: in the next section we presents the observation problem under sampling for a class of linear switching systems. In Section 3, we shall recall the technique of compressive sensing. In Section 4, we give a sufficient condition for the detection and reconstruction of active modes for a hybrid system. Section is devoted to the synthesis of impulsive observers. Finally, an academic example is proposed in order to prove the validity of the obtained results.

\section{PRoblem STATEMENT}

Consider a family of $r$ linear time invariant subsystems with discrete output measurements:

$$
\left\{\begin{array}{c}
\dot{z}^{i}(t)=\tilde{A}_{i} z^{i}(t) \\
y^{i}\left(t_{k}\right)=\tilde{C} z^{i}\left(t_{k}\right)
\end{array}\right.
$$

where $z^{i}(t) \in \mathbb{R}^{s}$ is the state vector associated with $i_{t h}$ subsystem, such that $z^{i}=\left(z_{1}^{i}, z_{2}^{i}, \cdots, z_{s}^{i}\right)^{T}$ with $i=\{1, \cdots, r\}$, $y_{\tilde{A}}^{i} \in \mathbb{R}^{p}$ represents the output vector measured in discrete time. $\tilde{A}_{i} \in \mathbb{R}^{s \times s}, \tilde{C} \in \mathbb{R}^{p \times s}$ are constant matrices with appropriate dimensions, and $p<s$.

Note that the system (1) can be considered as a hybrid dynamical system and more specifically as a switching system with a switching law and with resets functions equal to identity. The pairs $\left[\tilde{A}_{i}, \tilde{C}_{i}\right]$ are assumed to be observable.

Assumption 1. We assume that to each time interval $\Omega_{l}$ (called dwell time in each mode) there is a single subsystem 
that is active, and that the states of all other subsystems are equal to zero.

Assumption 2. We assume that during each time interval $\Omega_{l}$ there is at least $2 s+1$ output measurements $t_{k} \in T=$ $\left\{t_{0}, t_{1}, \ldots, t_{m}\right\}$, that are sparse ${ }^{1}$ and these are taken in a random fashion and are defined as follows:

There exists $\tau_{\min }$ and $\tau_{\max }$ with $0<\tau_{\min }<\tau_{\max }$ such that:

$$
\forall i>0: t_{i+1} \geq t_{i}+\tau_{\min } \text { and } t_{i+1} \leq t_{i}+\tau_{\max }
$$

By convention $t_{0}=0$.

The main objective of this paper is to bring a solution to the following question: Given a set of systems (1) satisfying the above assumptions, is it possible to detect the active system and rebuild the states of the system?

The problem consists, first of all, in the determination of sufficient conditions on the matrices $\tilde{A}_{i}$ and on the sampling periods $t_{k}$ such that the observability matrix (which is defined later in this paper) satisfies restrictive isometric property (RIP). Next, it will be shown that under these conditions that it is possible to synthesize an observer which permits observe the active state of the system and determine the associated active subsystem. The main contribution of this paper is to define

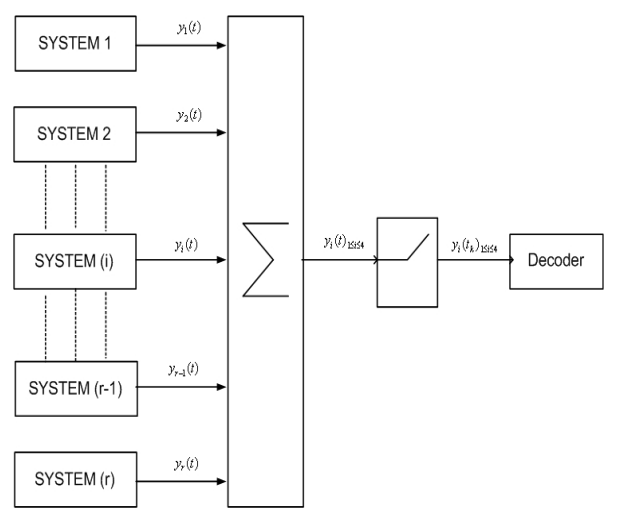

Fig. 1. block diagram of the studied system

observability conditions under sparse measurements and to link these conditions to the restricted isometry property (RIP) for compressive sensing [4].

Another contribution of this work is to propose a new impulsive observer scheme. This will allow to reconstruct the non-measured states even if they are unstable; this may be seen as the dual of reconstruction algorithms for compressive sensing such as the regularized scattering and linear inversion [9].

The necessary property for the solution to the problem is the property of strong distinguishability defined as follows:

Definition 1. The dynamical system (1) is said to be strongly distinguishable if $\forall i, j \in\{1, \cdots, r\}$, the matrices $\tilde{A}_{i}$ and $\tilde{A}_{j}$ have no common eigenvalues ${ }^{2}$.

\footnotetext{
${ }^{1}$ below Shanon-Nyquist frequency

${ }^{2}$ The random choice of $t_{k}$ avoid the pathological sampling see for example
}

\section{SOME FUNDAMENTAL CONCEPTS OF CS}

In this section, we recall some fundamental concept of compressive sampling namely the restricted isometry property (RIP) and reconstruction algorithms of signals under sparse measurements.

Definition 2. A signal $x(t)$ under $n$ sampling generates $a$ vector $X$ of $R^{n}$; that is $X=\left(x\left(t_{1}\right), \ldots, x\left(t_{n}\right)\right)^{T}$. The vector $X$ is said to be sparse is most of its components are zero; that is its support $\operatorname{supp}(x)=\left\{t_{1} \leq t_{k} \leq t_{n} \mid x\left(t_{k}\right) \neq 0\right\}$ is of cardinality $\|X\|_{0} \triangleq|\operatorname{supp}(x)|=s \ll n$.

Compressive sensing (CS) is a signal processing technique which renews the Shannon-Nyquist vision for sparse signals.

The theory of (CS) states that a signal can be reconstructed from $2 s+1$ measurements well below the current dimension $n$ via a linear projection; that is there exists a matrix $\Phi$ such that: $Y=\Phi X$, where $\Phi \in \mathbb{R}^{m \times n}$ is called measurement matrix with $2 s+1 \ll n$.

In general, it is not always possible to reconstruct by inversion the unknown signal $X$ from measurements $Y$ with dimension smaller than that of $X$.

However, if the input signal $X$ is sufficiently sparse and the matrix $\Phi$ satisfies the RIP of order two for a sufficiently small isometric constant $\delta_{s}$, then it is possible to uniquely reconstruct a $s$-sparse signal $X$ from measurements $Y$ by using a convex optimization program called " $l_{1}$-minimization" [5], [25].

The (RIP) also ensures that the recovery process is robust to noise [3]. Similar approaches in signal processing theory can be used for the reconstruction of signals with the aid of different iterative algorithms [21]. Before going back to control theory and in particular to the synthesis of observers, we recall RIP property of order $s$ :

Definition 3. Let $\phi \in \mathbb{R}^{m \times n}$, then $\phi$ is said to satisfy the RIP property of order $s$ if: there exists a constant $\left.\delta_{s} \in\right] 0 \quad 1[, \forall x$ such that $\|x\|_{0} \leq s$, we have:

$$
\left(1-\delta_{s}\right)\|x\|_{2}^{2} \leq\|\Phi x\|_{2}^{2} \leq\left(1+\delta_{s}\right)\|x\|_{2}^{2}
$$

Note that the RIP is an inherent property of the matrix $\Phi$. However, proving, in a deterministic fashion, that $\Phi$ verifies the RIP is very difficult, the computation is impossible when $n$ is very large. In addition, a major problem of the theory of compressive sensing is that it is difficult to practically build and to verify matrices satisfying the RIP. In fact, we do not know any deterministic and generic construction methods producing good measurement matrices (see [23] for an approach by chaotic systems). However, some random matrices in a well-chosen class verifies, with a high probability, the RIP property (see [15],[19]).

\section{CONDITION FOR THE DETECTION AND THE RECONSTRUCTION OF THE ACTIVE MODE}

In this section, we propose a new approach to derive the observability conditions under sampling and the detection of the active mode based on the technique of compressive sensing 
as described in the previous section. Let us consider the family of $r$ sub-systems defined previously by (1).

Assumption 3. Assume that the matrices $\tilde{A}_{i}$, with $\underset{\tilde{A}}{i}=$ $\{1, \cdots, r\}$ are strongly distinguishable and all couples $\tilde{A}_{i}, C$ are observable.

This assumption makes it always possible to ensure the existence of invertible transformation matrices $T_{i}$ such that they transform the system (1) (using the change of coordinates $X^{i}=T_{i} z^{i}$ ) in:

$$
\left\{\begin{array}{l}
\dot{X}^{i}(t)=A_{i} X^{i}(t) \\
y^{i}\left(t_{k}\right)=C X^{i}\left(t_{k}\right)
\end{array}\right.
$$

where the matrices $A_{i}$ are diagonal and they all have $s$ distinct eigenvalues $\lambda_{j}^{i}$, where $\lambda_{j}^{i}$ is the $j^{\text {th }}$ eigenvalue of the $i^{\text {th }}$ subsystem.

Without loss of generality, we suppose that output vector $y^{i}\left(t_{k}\right) \in \mathbb{R}^{p}$, and $C=(1,1, \cdots, 1)^{3}$, then we obtain:

$$
\left\{\begin{aligned}
y^{i}\left(t_{1}\right) & =C X^{i}\left(t_{1}\right)=C e^{A_{i} t_{1}} X^{i}\left(t_{0}\right) \\
y^{i}\left(t_{2}\right) & =C X^{i}\left(t_{2}\right)=C e^{A_{i} t_{2}} X^{i}\left(t_{0}\right) \\
\vdots & \\
y^{i}\left(t_{m}\right)=C X^{i}\left(t_{m}\right) & =C e^{A_{i} t_{m}} X^{i}\left(t_{0}\right)
\end{aligned}\right.
$$

which can be written in matrix form as follows:

$$
\begin{gathered}
Y=\Phi X\left(t_{0}\right) \\
\text { with } Y\left(t_{k}\right)=\left(\begin{array}{c}
y^{1}\left(t_{k}\right) \\
y^{2}\left(t_{k}\right) \\
\vdots \\
y^{r}\left(t_{k}\right)
\end{array}\right), X\left(t_{0}\right)=\left(\begin{array}{lllll}
X^{1}(0) \\
X^{2}(0) \\
\vdots \\
X^{r}(0)
\end{array}\right) \text { and } \\
\Phi=\left(\begin{array}{lllllll}
e^{\lambda_{1}^{1} t_{1}} & \cdots & e^{\lambda_{s}^{1} t_{1}} & \cdots & e^{\lambda_{1}^{r} t_{1}} & \cdots & e^{\lambda_{s}^{r} t_{1}} \\
\vdots & \vdots & \vdots & \vdots & \vdots & \vdots & \\
e^{\lambda_{1}^{1} t_{k}} & \cdots & e^{\lambda_{s}^{1} t_{k}} & \cdots & e^{\lambda_{1}^{r} t_{k}} & \cdots & e^{\lambda_{s}^{r} t_{k}} \\
e^{\lambda_{1}^{1} t_{k+1}} & \cdots & e^{\lambda_{s}^{1} t_{k+1}} & \cdots & e^{\lambda_{1}^{r} t_{k+1}} & \cdots & e^{\lambda_{s}^{r} t_{k+1}} \\
\vdots & \vdots & \vdots & \vdots & \vdots & \vdots & \vdots \\
e^{\lambda_{1}^{1} t_{m}} & \cdots & e^{\lambda_{s}^{1} t_{m}} & \cdots & e^{\lambda_{1}^{r} t_{m}} & \cdots & e^{\lambda_{s}^{r} t_{m}}
\end{array}\right)
\end{gathered}
$$

where $Y \in \mathbb{R}^{m}, X\left(t_{0}\right) \in \mathbb{R}^{n}$ is $s$-sparse and $\Phi \in \mathbb{R}^{m \times n}$

The observation problem related to the equation (5) thus reduces to a problem compressive sensing.

Proposition 1. Under the assumptions (1, 2 and 3), the active subsystem and the active state (1) can be reconstructed.

Remark 1. An almost similar result has been demonstrated for linear time-discrete systems in [8], [20], [24].

The proof of the proposition amounts in showing that any linear combination of $s$ column vectors of the matrix $\Phi$ is of full ranked. Denote by $\alpha_{j}:=\lambda_{i}^{j}, j=\{1, \ldots, s\}$ the eigenvalues corresponding to the chosen column vectors.

Without loss of generality, we consider the first $s$ column vectors of the matrix $\Phi$ (we can take other columns) and we

\footnotetext{
${ }^{3}$ due to the observability conditions and the fact that these matrices are diagonal none of the entries of the vector $C$ are equal to zero and it is always possible to determine a change of coordinates such that $C=(1,1, \cdots, 1)$.
}

denote:

$$
\Phi_{s}=\left(\begin{array}{cccc}
e^{\alpha_{1} t_{1}} & e^{\alpha_{2} t_{1}} & \cdots & e^{\alpha_{s} t_{1}} \\
e^{\alpha_{1} t_{2}} & e^{\alpha_{2} t_{2}} & \cdots & e^{\alpha_{s} t_{2}} \\
\vdots & \vdots & \vdots & \vdots \\
e^{\alpha_{1} t_{s}} & e^{\alpha_{2} t_{s}} & \cdots & e^{\alpha_{s} t_{s}}
\end{array}\right)
$$

The matrix $\Phi_{s}$ is exactly the generalized Vandermonde matrix [7] and its determinant is defined as follows:

$$
\operatorname{det} \Phi_{s}=e^{\alpha_{1} t_{1}} \prod_{k=2}^{s} e^{\alpha_{k} t_{1}} \Upsilon_{k} \quad \forall s \geq 2
$$

where $\Upsilon_{k}$ is recursively defined for $k>1$ as follows:

$$
\Gamma_{2}=\Upsilon_{2}=e^{\alpha_{2}\left(t_{2}-t_{1}\right)}-e^{\alpha_{1}\left(t_{2}-t_{1}\right)}
$$

and for $k \geq 3$, we have :

$\Upsilon_{k}=S_{\alpha_{k-1} \rightarrow \alpha_{k}}^{t_{k-1} \rightarrow t_{k}} \Upsilon_{k-1}-\left(S^{t_{k-1} \rightarrow t_{k}} \Upsilon_{k-1}\right) x \frac{S_{\alpha_{k-1} \rightarrow \alpha_{k}} \Gamma_{k-1}}{\Gamma_{k-1}}$

with :

$$
\begin{aligned}
\Gamma_{k}= & \left(S_{\alpha_{k-1} \rightarrow \alpha_{k}}^{t_{k-1} \rightarrow t_{k}} \Gamma_{k-1}\right) \Gamma_{k-1}-S_{\alpha_{k-1} \rightarrow \alpha_{k}} \Gamma_{k-1} S^{t_{k-1} \rightarrow t_{k}} \Gamma_{k-1} \\
& S^{t_{k-1} \rightarrow t_{k}} \Gamma_{k-1} \text { is the permutation } t_{k} \text { by } t_{k-1} \text { in } \Gamma_{k-1} \\
& S_{\alpha_{k-1} \rightarrow \alpha_{k}} \Gamma_{k-1} \text { is the permutation } \alpha_{k-1} \text { by } \alpha_{k} \text { in } \Gamma_{k-1}
\end{aligned}
$$

To show that the matrix $\Phi_{s}$ is full rank, it is sufficient to show $\operatorname{det} \Phi_{s} \neq 0$ as, which is the same as showing that all $\Upsilon_{k}$ are nonzero. We distinguish two cases:

Case 1: All the eigenvalues of the matrix $\Phi_{s}$ are real.

Since the subsystems are highly distinguishable and observable, then all the eigenvalues of the matrix $\Phi_{s}$ are distinct and non-zero, and therefore $\Phi_{s}$ matrix is full rank, hence confirming the result.

Case 2: The eigenvalues of the matrix $\Phi_{s}$ are complex.

In this case, we first show by induction that the $\Gamma_{k}$ are nonzero for all $k=\{1, \cdots, m\}$ and for all $m \in \mathbb{N}$ :

For $k=2$, we have:

$$
\Gamma_{2}=e^{\alpha_{2}\left(t_{2}-t_{1}\right)}-e^{\alpha_{1}\left(t_{2}-t_{1}\right)}
$$

As $\alpha_{1} \neq \alpha_{2}$, then $e^{\alpha_{2}\left(t_{2}-t_{1}\right)} \neq e^{\alpha_{1}\left(t_{2}-t_{1}\right)}$ except in the case where $\alpha_{1}$ and $\alpha_{2}$ are purely imaginary. But note that even if $e^{\alpha_{2}\left(t_{2}-t_{1}\right)}=e^{\alpha_{1}\left(t_{2}-t_{1}\right)}$, the fact we have $2 s+1$ instant choices and that these measurement instants are randomly chosen, then it is always possible to have other eigenvalues such that $\Gamma_{2} \neq 0$ (by choosing other columns of the matrix $\Phi$ ), and hence the probability of having $\Gamma_{2}=0$ is zero.

For $k \geq 3$, suppose that: $\Gamma_{k-1} \neq 0$ for all $k \geq 3$ arbitrary and fixed in $\mathbb{N}$ is verified. The conditions of assumptions 2 and 3 , we have $\alpha_{k} \neq \alpha_{j}$ for all $j \in\{1, \ldots, k-1\}$ and $t_{k}>t_{k-1}>\ldots>t_{1}$, which implies in a probabilistic fashion that the following permutations $S_{\alpha_{k-1} \rightarrow \alpha_{k}} \Gamma_{k-1}$, $S^{t_{k-1} \rightarrow t_{k}} \Gamma_{k-1}$ and $S_{\alpha_{k-1} \rightarrow \alpha_{k}}^{t_{k-1} \rightarrow t_{k}} \Gamma_{k-1}$ are non zero and similarly for $\left(S_{\alpha_{k-1} \rightarrow \alpha_{k}}^{t_{k-1} \rightarrow t_{k}} \Gamma_{k-1}\right) \Gamma_{k-1} \neq 0$.

On the other hand, we have :

$\Gamma_{k}=\left(S_{\alpha_{k-1} \rightarrow \alpha_{k}}^{t_{k-1} \rightarrow t_{k}} \Gamma_{k-1}\right) \Gamma_{k-1}-S_{\alpha_{k-1} \rightarrow \alpha_{k}} \Gamma_{k-1} S^{t_{k-1} \rightarrow t_{k}} \Gamma_{k-1}$

Hence, the only possibility so that $\Gamma_{k}=0$ is:

$$
\left(S_{\alpha_{k-1} \rightarrow \alpha_{k}}^{t_{k-1} \rightarrow t_{k}} \Gamma_{k-1}\right) \Gamma_{k-1}=S_{\alpha_{k-1} \rightarrow \alpha_{k}} \Gamma_{k-1} S^{t_{k-1} \rightarrow t_{k}} \Gamma_{k-1}
$$


while, $\alpha_{k} \neq \alpha_{j}, \forall j \in\{1, \ldots, k-1\}$, and $t_{k}>t_{k-1}>\ldots>$ $t_{1}$. Owing to the fact that we have at least $2 s+1$ choices of these measurement instances, the probability of having the equality (8) is practically zero (by choosing other columns of the matrix $\Phi$ which renders the equality (8) false). The same reasoning is used to show that $\Upsilon_{k} \neq 0$.

\section{IMPULSIVE OBSERVER DESIGN}

In this section, an impulsive observer is proposed, it allows estimate the states of each subsystem defined by (1) using only sampled outputs measurements. First, the case of linear systems where all unstable states are measured is considered. Afterwards, the case of linear systems with non-measured unstable dynamics are studied. Consider the following linear system:

$$
\left\{\begin{array}{l}
x_{1}(t)=A_{11} x_{1}(t)+A_{12} x_{2}(t) \\
x_{2}(t)=A_{21} x_{1}(t)+A_{22} x_{2}(t) \\
y\left(t_{k}\right)=x_{1}\left(t_{k}\right)
\end{array}\right.
$$

where $x(t)=\left(x_{1}^{T}(t), x_{2}^{T}(t)\right) \in \mathbb{R}^{s}$ with $A_{11} \in \mathbb{R}^{p \times p}, A_{12} \in$ $\mathbb{R}^{p \times(s-p)}, A_{21} \in \mathbb{R}^{(s-p) \times(s)} A_{22} \in \mathbb{R}^{(s-p) \times(s-p)}$.

\section{A. Case 1: The unstable states are measured}

Assumption 4. Assume that $K e r C \subset \operatorname{span}\left\{E^{s}\right\}$, where $E^{s}$ is a stable manifold of the system (9).

Remark 1. Hypothesis 4 signifies that the number of outputs must be at least equal to the number of unstable directions. This condition is consistent to the Pyragas conjecture [18] in the theory of control which states that one must have as many outputs as unstable Lyapunov exponents in order to be able to estimate the systems states.

Under assumption (4) the proposed impulsive observer corresponding to system (9) is constructed as follows:

$$
\left\{\begin{array}{l}
\dot{\hat{x}}_{1}(t)=A_{11} \hat{x}_{1}(t)+A_{12} \hat{x}_{2}(t) \\
\dot{\hat{x}}_{2}(t)=A_{21} \hat{x}_{1}(t)+A_{22} \hat{x}_{2}(t) \\
\hat{x}_{1}\left(t_{k}^{+}\right)=R \hat{x}_{1}\left(t_{k}\right)+\left(I_{d}-R\right) x_{1}\left(t_{k}\right)
\end{array}\right.
$$

where $R=\operatorname{diag}\left\{r_{1}, . ., r_{p}\right\}$ with $-1<r_{i}<1$, for $i=1, \ldots, p$.

Note that system (10) has the same continuous dynamic as system (9), except that at each measurement instant of the state, the observer is reset or reinitialized as a function of the error between the estimated output $\hat{y}\left(t_{k}\right)$ and the measured output $y\left(t_{k}\right)$.

Proposition 2. [14] Under assumption 4 and for bounded sampling periods $\theta_{k}$, it is always possible to design an observer of the type (10) which converges to the states of system (9).

Remark 2. The observability or detectability condition is not sufficient for the synthesis of this type of impulsive observer as the output here are discrete. One must add a condition on the unstable states that are measured. In the next section, this condition is relaxed.

\section{B. Case 2: Some unstables state are not measured}

Consider again system (9) defined above with:

- $x_{1} \in \mathbb{R}^{p}$ are the measured unstable or stable states.

- $x_{2} \in \mathbb{R}^{s-p}$ are unmeasured states are unstable or stable, but at least detectable.

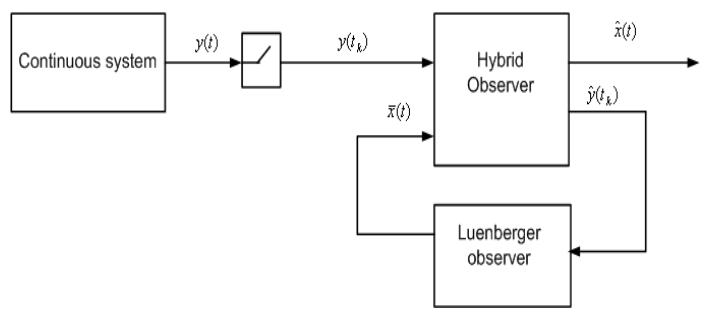

Fig. 2. Generalized impulsive observer

The proposed generalized impulse observer (see Figure 2) is designed as follows:

$$
\left\{\begin{array}{l}
\dot{\hat{x}}_{1}(t)=A_{11} \hat{x}_{1}(t)+A_{12} \hat{x}_{2}(t) \\
\dot{\hat{x}}_{2}(t)=A_{22} \hat{x}_{2}(t)+M\left(\tilde{x}_{2}(t)-\hat{x}_{2}(t)\right) \\
\dot{\tilde{x}}_{1}(t)=A_{11} \tilde{x}_{1}(t)+A_{12} \tilde{x}_{2}(t)+L_{1}\left(\hat{x}_{1}(t)-\tilde{x}_{1}(t)\right) \\
\dot{\tilde{x}}_{2}(t)=A_{22} \tilde{x}_{2}(t)+L_{2}\left(\hat{x}_{1}(t)-\tilde{x}_{1}(t)\right) \\
\hat{x}_{1}\left(t_{k}^{+}\right)=R \hat{x}_{1}\left(t_{k}\right)+\left(I_{d}-R\right) x_{1}\left(t_{k}\right)
\end{array}\right.
$$

where $R=\operatorname{diag}\left\{r_{1}, . ., r_{p}\right\}$ and $-1<r_{i}<1$, for $i=\{1, \ldots, p\} . R, M, L_{1}$ et $L_{2}$ are constant matrices with appropriate dimensions.

The observation error $\chi \triangleq\left(e_{i}, \bar{e}_{i}\right)$ with $e_{i}=x_{i}-\hat{x}_{i}$ and $\bar{e}_{i}=x_{i}-\tilde{x}_{i}$ is:

$$
\left\{\begin{array}{l}
\dot{e}_{1}(t)=A_{11} e_{1}(t)+A_{12} e_{2}(t) \\
\dot{e}_{2}(t)=A_{22} e_{2}(t)+M \bar{e}_{2}(t)-M e_{2} \\
\dot{\bar{e}}_{1}(t)=A_{11} \bar{e}_{1}(t)+A_{12} \bar{e}_{2}(t)-L_{1} \bar{e}_{1}(t)+L_{1} e_{1} \\
\dot{\bar{e}}_{2}(t)=A_{22} \bar{e}_{2}(t)-L_{2} \bar{e}_{1}(t)+L_{2} e_{1} \\
e_{1}\left(t_{k}^{+}\right)=R_{1}\left(t_{k}\right)
\end{array}\right.
$$

Proposition 3. [13] Given the system (9) and the observer (11), then $\forall \epsilon>0$, there exists $\beta_{\max }>\epsilon$, a maximum sampling period $\theta_{\max }>0$ and an reset matrix $R$, such that for every initial condition $\|x(0)\|<\beta_{\max }$, the observation error (12) converges to a ball of radius $\epsilon+\epsilon^{*}$.

Set $\chi_{1}:=e_{1}$ and $\chi_{2}:=\left(e_{2}^{T}, \bar{e}_{1}^{T}, \bar{e}_{2}^{T}\right)^{T}$. The observation error takes the following form:

$$
\left\{\begin{array}{l}
\dot{\chi}_{1}(t)=\bar{A}_{11} \chi_{1}(t)+\bar{A}_{12} \chi_{2}(t) \\
\dot{\chi}_{2}(t)=\bar{A}_{21} e_{2}(t)+\bar{A}_{22} \chi_{2}(t) \\
\left.\chi_{1}\left(t_{k}^{+}\right)=R \chi_{(} t_{k}\right)
\end{array}\right.
$$

with $\bar{A}_{11}=A_{11}, \bar{A}_{12}=\left(\begin{array}{ccc}A_{12} & 0 & 0\end{array}\right)$,

$$
\bar{A}_{21}=\left(\begin{array}{c}
0 \\
L_{1} \\
L_{2}
\end{array}\right) \bar{A}_{22}=\left(\begin{array}{ccc}
A_{22}-M & 0 & M \\
0 & A_{11}-L_{1} & A_{12} \\
0 & -L_{2} & A_{22}
\end{array}\right)
$$

Define the Lyapunov fonction:

$V_{1}\left(\chi_{1}(t)\right)=\left\|\chi_{1}(t)\right\|_{1}$ and $V_{2}\left(\chi_{2}(t)\right) \triangleq \chi_{2}^{T}(t) P \chi_{2}(t)$ with $\|\cdot\|_{1}$ is one-norm and $P$ is a positive definite symmetric matrix.

$$
\begin{aligned}
V_{1}\left(\chi_{1}\left(t_{k+1}^{+}\right)\right) & \leq\left\|R e^{\bar{A}_{11} \theta_{k}} \chi_{1}\left(t_{k}\right)\right\|_{1} \\
& +\int_{t_{k}}^{t_{k+1}}\left\|R e^{\bar{A}_{11}\left(t_{k+1}-\tau\right)} \bar{A}_{12} \chi_{2}(\tau)\right\|_{1} d \tau
\end{aligned}
$$


suppose that $\forall \tau \in\left[t_{k}^{+}, t_{k+1}\right]:\left\|\chi_{2}(\tau)\right\|<\beta$ (this will be shown subsequently), we obtain:

$$
\begin{aligned}
V_{1}\left(\chi_{1}\left(t_{k+1}^{+}\right)\right) & \leq\left\|R e^{\bar{A}_{11} \theta_{k}} \chi_{1}\left(t_{k}\right)\right\|_{1} \\
& +\|R\|_{1}\left\|\bar{A}_{12}\right\|_{1} \beta \int_{t_{k}}^{t_{k+1}}\left\|e^{\bar{A}_{11}\left(t_{k+1}-\tau\right)}\right\|_{1} d \tau
\end{aligned}
$$

with $V_{1, \text { Max }} \triangleq \max _{\left\|\chi_{1}\right\|=\beta_{\max }}\left\{V_{1}\left(\chi_{1}\right)\right\}$, then $\forall \epsilon>0$ sufficiently small, it is always possible to find $R$ such that, $V_{1}\left(\chi_{1}\left(t_{k+1}^{+}\right)\right)<\epsilon$, we obtain

$$
\left\|\chi_{1}\left(t_{k+1}\right)\right\|<\epsilon
$$

On the other hand, for $V_{2}\left(\chi_{2}(t)\right)$, we have:

$$
\begin{aligned}
\dot{V}_{2}\left(\chi_{2}(t)\right) & =\chi_{2}^{T}(t)\left(\bar{A}_{22}^{T} P+P \bar{A}_{22}\right) \chi_{2}(t) \\
& +2\left\|\chi_{1}^{T}(t) \bar{A}_{21} P \chi_{2}(t)\right\|
\end{aligned}
$$

According to the structure of the matrix $\bar{A}_{22}$, it is always possible to find gains observation $M, L_{1}$ and $L_{2}$ that make a Hurwitz matrix. then there exists a positive definite symmetric matrix $Q$ such that $\bar{A}_{22}^{T} P+P \bar{A}_{22}=-Q$. Thus

$$
\dot{V}_{2}(t)=-\chi_{2}^{T}(t) Q \chi_{2}(t)+2\left\|\chi_{1}^{T}(t) \bar{A}_{21} \chi_{2}(t)\right\|
$$

but $\chi_{2}^{T}(t) Q \chi_{2} \geq \lambda_{\min }(Q)\left\|\chi_{2}(t)\right\|^{2}$ where $\lambda_{\min }(Q)$ denote the minimum eigenvalue of a matrix and it is real and positive since $Q$ is positive definite symmetric matrix. Thus

$$
\dot{V}_{2}(t) \leq\left(-\lambda_{\min }(Q)\left\|\chi_{2}(t)\right\|+2\left\|\chi_{1}^{T}(t) \bar{A}_{21}\right\|\right)\left\|\chi_{2}(t)\right\|
$$

suppose that $\left\|\chi_{2}\right\| \geq \epsilon^{*}$ with

$$
\epsilon^{*}=\frac{2 \epsilon\left\|\bar{A}_{21} P\right\|}{\lambda_{\min }(Q)}
$$

which gives us:

$$
\dot{V}_{2}\left(\chi_{2}(t)\right)<0
$$

Donc $\chi_{1}$ converge toward a ball of radius $\epsilon$ and $\chi_{2}$ converge toward a ball of radius $\epsilon^{*}$.

hence $\chi$ converge toward a ball of radius $\epsilon+\epsilon^{*}$.

This completes the proof.

\section{Design of Multi-ObServer}

Generally, the choice of bases in compressive sensing is very important task (Fourier analysis, wavelets [22]) in signal processing. Similarly, some information (for example the regrouping nonzero information) on the signal are very useful for the design of a decompression algorithm. Thus, in control theory, one of the main question with the aim to elaborate a method inspired by the technique compressive sensing is: what is the appropriate basis? The works of Poincar [17] on normal forms for the study the stability of dynamical systems in the theory of ordinary differential equation has been extended to control theory by W. Kang and A. Krener [12] in order to study the controllability dynamical systems.

In addition, some authors have proposed normal forms for the study of observability [26], from these work. It is therefore natural to investigate whether the normal observability forms can be an appropriate basis for the observation of the system's state under sparse measurement.

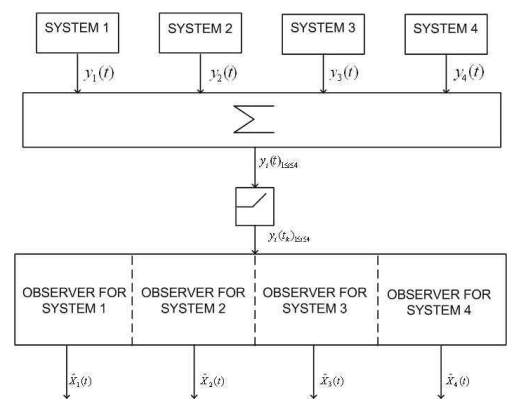

Fig. 3. Multi-observer bloc diagram

Nevertheless, the main objective here is to detect the subsystem which is in active mode and to reconstruct its corresponding state. Finally we use a technique called the Multi-observer [1], [16] (see Figure 3) where the detection of active mode is determined by a threshold of each residual (dynamic error observation).

In fact, for each subsystem, we design an impulsive subobserver that converges toward the corresponding state of the system. These sub-observer receive sparse measurements, the observation error $e_{i}=y-\hat{y}^{i}, i=1, \ldots r$ between measured output and each sub-observer allows to detect the system that is in active mode.

\section{Simulation RESUltS}

Here we present academic example of linear switching system. Consider a set of four sub-linear systems as follows:

$$
\begin{gathered}
\Sigma_{1}=\left\{\begin{array}{l}
\dot{x}_{1}^{1}=3.5 x_{1}^{1}-4 x_{2}^{1} \\
\dot{x}_{2}^{1}=5.5 x_{1}^{1}-3.5 x_{2}^{1}
\end{array}\right. \\
\Sigma_{2}=\left\{\begin{array}{l}
\dot{x}_{1}^{2}=2 x_{1}^{2}-3 x_{2}^{2} \\
\dot{x}_{2}^{2}=3 x_{1}^{2}-2 x_{2}^{2}
\end{array}\right. \\
\Sigma_{3}=\left\{\begin{array}{l}
\dot{x}_{1}^{3}=1.5 x_{1}^{3}+3 x_{2}^{3} \\
\dot{x}_{2}^{3}=-2 x_{1}^{3}-1.5 x_{2}^{3}
\end{array}\right. \\
\Sigma_{4}=\left\{\begin{array}{l}
\dot{x}_{1}^{4}=0.5 x_{1}^{4}+2 x_{2}^{4} \\
\dot{x}_{2}^{4}=-x_{1}^{4}-0.5 x_{2}^{4}
\end{array}\right.
\end{gathered}
$$

where $\left(x_{1}^{i}, x_{2}^{i}\right) \in \mathbb{R}^{2}, i=\{1,2,3,4\}$ and $y\left(t_{k}\right)=\sum_{i=1}^{4} x_{1}^{i}\left(t_{k}\right)$ with $t_{k} \in[0.1,0.5]$. It is assumed that there exists only one system that is active at each dwell time $\tau_{i}=50 \mathrm{~s}$.

The eigenvalues of the four subsystems are respectively: $\lambda_{1}^{1}=3.1225 i, \lambda_{2}^{1}=-3.225 i, \lambda_{1}^{2}=2.236, \lambda_{2}^{2}=-2.236, \lambda_{1}^{3}=$ $1.9365 i, \lambda_{2}^{3}=61.9365 i, \lambda_{1}^{4}=1.3229 i$ and $\lambda_{2}^{4}=-1.3229 i$.

The behavior of the considered hybrid system is represented in Figure 4; In order to rebuild the states and to detect the active subsystem, we propose a multi-observer containing four sub-observers such that each sub-observer converges to the corresponding sub-system if the latter is active; that is:

$$
O B S_{1}=\left\{\begin{array}{l}
\dot{\hat{x}}_{1}^{1}=3.5 \hat{x}_{1}^{1}-4 \hat{x}_{2}^{1} \\
\dot{\hat{x}}_{2}^{1}=5.5 \hat{x}_{1}^{1}-3.5 \hat{x}_{2}^{1} \\
\hat{x}_{1}^{1}\left(t_{k}^{+}\right)=y\left(t_{k}\right)
\end{array}\right.
$$



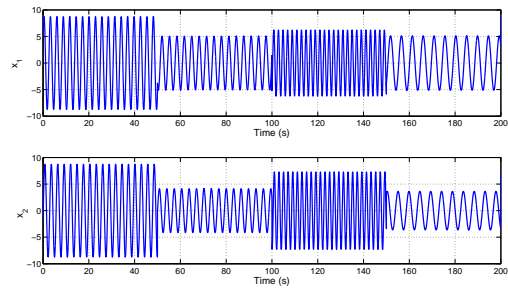

Fig. 4. stats $x_{1}(t)$ and $x_{2}(t)$

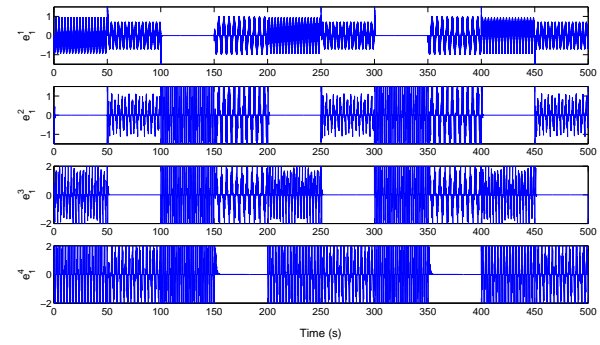

Fig. 5. Observation error of each sub-system with respect to the measured output

$$
\begin{gathered}
O B S_{2}=\left\{\begin{array}{l}
\dot{\hat{x}}_{1}^{2}=2 \hat{x}_{1}^{2}-3 \hat{x}_{2}^{2} \\
\dot{x}_{2}^{2}=3 \hat{x}_{1}^{2}-2 \hat{x}_{2}^{2} \\
\hat{x}_{1}^{2}\left(t_{k}^{+}\right)=y\left(t_{k}\right)
\end{array}\right. \\
O B S_{3}=\left\{\begin{array}{l}
\dot{\hat{x}}_{1}^{3}=1.5 \hat{x}_{1}^{3}+3 \hat{x}_{2}^{3} \\
\dot{x}_{2}^{3}=-2 \hat{x}_{1}^{3}-1.5 \hat{x}_{2}^{3} \\
\hat{x}_{1}^{3}\left(t_{k}^{+}\right)=y\left(t_{k}\right)
\end{array}\right. \\
O B S_{4}=\left\{\begin{array}{l}
\dot{\hat{x}}_{1}^{4}=0.5 \hat{x}_{1}^{4}+2 \hat{x}_{2}^{4} \\
\dot{x}_{2}=-\hat{x}_{1}^{4}-0.5 \hat{x}_{2}^{4} \\
\hat{x}_{1}^{4}\left(t_{k}^{+}\right)=y\left(t_{k}\right)
\end{array}\right.
\end{gathered}
$$

It is shown that the observation error of each subsystem is stable. Figure 5 puts into evidence the efficiency of the proposed method and show respectively the convergence of each sub-observer to the subsystem to which it is associated when the latter is active. The Figure 5 shows the active trajectory. In addition, if the observation error is null for a subsystem, then we can confirm that it is active and vice versa. For example, during the time period $[0,50]$, we have $e_{1}^{2}(t)=0$, therefore it is sub-system 2 that is active, during the period $[250,300]$ we have $e_{1}^{3}(t)=0$, then it is subsystem 3 that is active.

\section{CONCLUSION}

In this paper, we have shown that it is possible to detect and reconstruct the state of the active mode for a class of linear switching systems with sparse measurments. A sufficient condition which can be seen as the dual of the RIP property for compressive sensing is given. Two types of observers have been designed: the first one, which is impulsive, for measured unstable states and the other, which is a generalized impulsive observer, for non-measured unstable states. The simulation results obtained using an impulsive multi-observer confirmed the good performance of the proposed schemes.

\section{REFERENCES}

[1] B. D. O. Anderson, T. S. Brinsmead, F. De Bruyne, J. Hespanha, D. Liberzon and A. S. Morse, Multiple Model Adaptive Control. I. Finite Controller Coverings, George Zames Special Issue, International Journal of Robust and Nonlinear Control, vol. 10, pp. 909-929, 2000.

[2] L. Berec, A multi-model method to fault detection and diagnosis: Bayesian solution. an introductory treatise, International Journal of Adaptive Control and Signal Processing, vol. 12, p. 8192, 1998.

[3] E. J. Candés, The restricted isometry property and its implications for compressed sensing, Compte Rendus de 1Academie des Sciences, Series 1, vol. 346, pp. $910,2008$.

[4] E. J. Candés and M. B.Wakin, An introduction to compressive sampling, IEEE Signal Processing Magazine, vol. 21, no. 2, pp. 2130, 2008.

[5] E. J. Candés and T. Tao, Decoding via linear programming, IEEE Transactions on Information Theory, vol. 51, no. 12,pp. 42034215, 2005.

[6] T. Chen, B. Francis, Optimal sampled-data control systems, Springer verlag, London, 1995.

[7] Y. Chen, H. Li and E. Tan , An Explicit Factorization Of Totally Positive Generalized Vandermonde Matrices Avoiding Schur Functions, Applied Mathematics E-Notes, 2008.

[8] W. Dai and S. Yuksel, Technical report: Observability of a linear system under sparsity constraints, ArXiv preprint arXiv:1204.3097, 2012.

[9] D. L. Donoho, Compressed sensing, IEEE Trans. Inf. Theory, vol. 52, no. 4, pp. 1289-1306, 2006.

[10] D. Donoho, I. Drori, V. Stoden, Y. Tsaig, and M. Shahram, Sparselab, http://sparselab.stanford.edu.

[11] J-P. Gauthier and I. Kupka, Deterministic observation theory and applications, Cambridge University Press, 2001.

[12] W. Kang and A. J. Krener, Extended quadratic controller normal form and dynamic state feedback linearization of nonlinear systems, SIAM J. Control and Optimization, vol. 30, pp. 13191337, 1992.

[13] Y. Khaled, J. P. Barbot et D. Benmerzouk Un nouveau type d'observateur impulsionnel, 2nd Confrence Internationale Francophone d'Automatique, Grenoble, 2012.

[14] Y. Khaled, J. P. Barbot, D. Benmerzouk, K. Busawon and M. Ghanes, Strange attractor identification and state observation under sparse measurements, 2nd International Symposium On Environment Friendly Energies And Applications, Northumbria. 2012.

[15] A. Mazumdar and A. Barg, General constructions of deterministic (s)rip matrices for compressive sampling, IEEE International Symposium on Information Theory (ISIT), pp. 678682, 2011.

[16] A. S. Morse, control of families of linear set-point controllers-part 1: Exact matching, IEEE Transactions on Automatic Control, vol. 41, pp. 1413-1431,October 1996.

[17] H. Poincar, Solutions priodiques dans le voisinage d.un point d.quilibrelunes sans quadrature, Les Mthodes Nouvelles de la Mcanique Cleste, Gauthier-Villars, pp. 156161, 1892.

[18] K. Pyragas, Predictable chaos in slightly perturbed unpredictable chaotic systems, Physics Letters, vol. 181, no. 3, pp. 203210, 1993.

[19] A. D. Ronald, Deterministic constructions of compressed sensing matrices, Journal of Complexity, vol. 23, no. 4-6, pp. 918925, 2007.

[20] B. M. Sanandaji, M. B. Wakin and T. L. Vincent, Observability with random observations, arXiv preprint arXiv: 1211.4077, 2012.

[21] J. A. Tropp and A. C. Gilbert, Signal recovery from random measurements via orthogonal matching pursuit, IEEE Transactions on Information Theory, vol. 53, no. 12, pp. 46554666, 2007.

[22] A. Woiselle, J. Starck, and M. Fadili, $3 d$ data denoising and inpainting with the fast curvelet transform, Journal of Mathematical Imaging and Vision (JMIV), vol. 39, no. 2, pp. 121139, 2011.

[23] L. Yu, J. P. Barbot, G. Zheng, and H. Sun, Toeplitz-structured Chaotic Sensing Matrix for Compressive Sensing, Communication Systems Networks and Digital Signal Processing (CSNDSP) Symp, pages 229-233, 2010 .

[24] M. B. Wakin, B. M. Sanandaji and T. L. Vincent, On the observability of linear systems from random compressive measurements, Decision and Control (CDC), 49th IEEE Conference on. IEEE, 2010.

[25] J. Zhao, B. Song, N. Xi, K. C. Lai, H. Chen and C. Qu, Compressive feedback based non-vector space control. In American Control Conference (ACC), pp. 4090-4095, 2012. 
[26] G. Zheng, D. Boutat, and J. P. Barbot, Single outputdependent observability normal form, SIAM, J. Control Optim, vol. 46, pp. 22422255 , 2007. 